\title{
Prevalence of galaxy-galaxy interactions in AGN hosts
}

\author{
Jeremy Lim ${ }^{1}$, Cheng-Yu Kuo ${ }^{1}$, Ya-Wen Tang ${ }^{1}$, Jenny Greene ${ }^{2}$ and \\ Paul T. P. Ho $^{2}$
${ }^{1}$ Institute of Astronomy \& Astrophysics, Academia Sinica, PO Box 23-141, Taiepei 106, Taiwan email: jlim@asiaa.sinica.edu.tw, cykuo@asiaa.sinica.edu.tw, ywtang@asiaa.sinica.ewdu.tw email: jgreene@cfa.harvard.edu, ho@cfa.harvard.edu \\ ${ }^{2}$ Harvard-Smithsonian Center for Astrophysics, 60 Garden Street, Cambridge, MA 02138, USA
}

\begin{abstract}
Studies in optical starlight have failed to reach a consensus on the importance of either galaxy interactions, bars, or nuclear spirals in triggering luminous active galactic nuclei (AGNs). Here, we present the first systematic imaging study of Seyfert (disk) galaxies in the 21-cm line of neutral atomic hydrogen (HI) gas. HI is the most sensitive and enduring tracer of galaxy interactions, and can reveal tidal features not otherwise visible in optical starlight. Our sample comprises all twenty-eight galaxies in the Véron-Cetty \& Véron (1998) catalog with nuclear magnitudes $-19 \geqslant M_{\mathrm{B}}>-23$ (including Seyfert, LINER, and HII galaxies) at $0.015 \leqslant z \leqslant 0.017$ in the northern hemisphere, and a matched control sample of twenty-seven inactive galaxies at $z \approx 0.008$. We have detected nearly all the galaxies observed, and find a much higher incidence of tidal interactions - usually not seen in optical starlight — among the Seyfert galaxies by comparison with the matched control sample. Those Seyferts with uncertain or no clear tidal features show disturbed HI morphologies and/or kinematics, as well as HI companion galaxies, more frequently than the control sample. Our study suggests that the undisturbed optical appearence of active galaxies may be deceptive, and imply that galaxygalaxy interactions trigger a significant fraction luminous AGNs at low redshifts. The majority of the Seyfert galaxies in our sample appear to be at a relatively early stage of an encounter rather than late in a merger.
\end{abstract}

\section{The Poster}

In the poster, we showed illustrative examples of our HI maps for both the Seyfert galaxies and matched control sample of inactive galaxies. Four of these maps are reproduced in Figure 1. Ark 539 (top row) is a Seyfert galaxy that does not appear to be disturbed in the optical. In HI, however, a tidal bridge can be seen connecting the southern part of the Seyfert galaxy to a companion galaxy (at the same redshift) to the south-east. A partial tidal bridge or tail is visible emerging from the northern part of the Seyfert galaxy. None of these features are visible in the optical. Markarian 993 also does not appear to be disturbed in the optical, and in HI is one of the least disturbed Seyfert galaxies in our sample. Nevertheless, in HI, it exhibits a protruberance on the north-east side pointing towards a companion galaxy, suggestive of a tidal feature. In between these two extremes, we found Seyfert galaxies that show HI asymmetries likely tracing tidal interactions with companion galaxies detected also in HI, or disturbed HI morphologies and/or kinematics that may be caused by interactions with companion galaxies again detected in HI. NGC 2543 (third row) is one of the inactive galaxies from our control sample, and the only one found, in HI, to be interacting with (two) companion galaxies. These HI tidal features are not visible in the optical. The remainder, like NGC 4814 (bottom row), show normal HI morphologies and kinematics. 


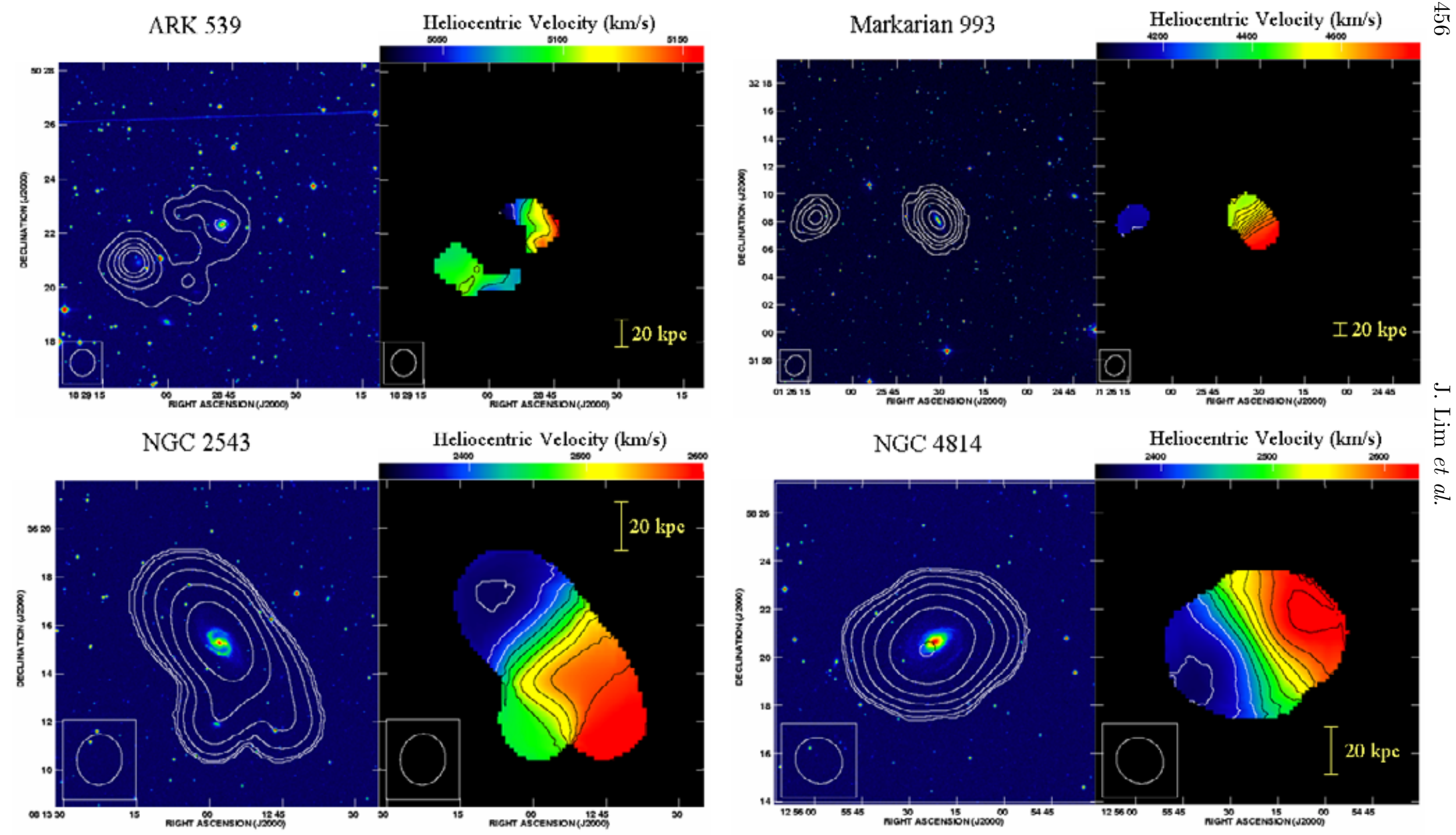

Figure 1. Left panels - contours of the integrated HI intensity overlaid on grayscale images of the optical fields (from the Digitized Sky Survey) centered on each target object. Right panels - contours and grayscale-coded images of the intensity-weighted mean HI velocity. The synthesized beam is shown in the lower left corner of each panel. The target objects in the upper two rows are from our sample of Seyfert galaxies, whereas those in the lower two rows are from our sample of matched inactive galaxies. The tidal features detected in HI are not visible in optical starlight. 